\title{
Posterior Reversible Encephalopathy Syndrome; An Unusual Manifestation of Systemic Lupus Erythematosus : A Case Report
}

\author{
MANIK KUMAR TALUKDER ${ }^{1}$, MD. IMNUL ISLAM ${ }^{1}$, SURAIYA BEGUM ${ }^{1}$, GOPEN KUMAR KUNDU ${ }^{1}$, \\ SHAHANAARAHMAN ${ }^{2}$
}

\begin{abstract}
Introduction
Posterior reversible encephalopathy syndrome (PRES) is a neurological condition diagnosed by clinical presentation and neuro-imaging findings ${ }^{1}$. With the advent of modern neuro-imaging techniques and awareness amongst physician, PRES has been increasingly identified since the condition was first described in $1996^{2}$. Some names have been proposed for this clinical entity, including reversible posterior cerebral edema, reversible posterior leucoencephalopathy syndrome, reversible occipitoparietal encephalopathy and hyperperfusion encephalopathy ${ }^{3}$. Majority of PRES cases reported were related to conditions, such as pre-eclampsia, eclampsia, hypertensive crisis, thrombotic thrombocytopenic purpura (TTP), post-chemotherapy and solid organ transplantation ${ }^{4}$. PRES is often recognized in patients with autoimmune conditions such as systemic lupus erythematosus (SLE) and systemic vasculitis ${ }^{5}$. SLE is an autoimmune disease characterized by its varied clinical manifestation with multi-system involvements ${ }^{6}$. Very few case reports are available among SLE patients presenting with PRES. We are reporting a case of SLE with PRES. It is important to recognize the condition early in order to treat the condition effectively and to minimize the morbidity and mortality.
\end{abstract}

\section{Case Report:}

' $N$ ', an 8 years old girl, $1^{\text {st }}$ issue of nonconsanguineous parents was admitted in the department of paediatrics, Bangabandhu Sheikh Mujib Medical University (BSMMU) on 08.122010 with the complaints of convulsion followed by unconsciousness for two days. She also had history of irregular fever, headache and weight loss for 2 months. ' $N$ ' had

1. Assistant Professor, Department of Paediatrics, Bangabandhu Sheikh Mujib Medical University, Dhaka, Bangladesh.

2. Professor, Department of Paediatrics, Bangabandhu Sheikh Mujib Medical University, Dhaka, Bangladesh.

Correspondence: Dr. Manik Kumar Talukder multiple joint pain and swelling for the same duration. She had no history of oral ulceration, skin or malar rash, photosensitivity, alopecia, dyspnoea, dysuria or chest pain. On examination, ' $\mathrm{N}$ ' was unconscious (Glasgo Coma Scale: 4) and mildly pale. Her pulses were normal, blood pressure was raised $(150 / 100 \mathrm{~mm}$ $\mathrm{Hg}$ ) and respiratory rate was $32 / \mathrm{min}$. Body surface area is $0.79 \mathrm{~m}^{2}$. Bedside urine showed heavy proteinuria $(+++)$. She had hepatomegaly $(4 \mathrm{~cm})$ which was firm and non tender. There was no splenomegaly and ascitis. Her muscle tone was increased, all the reflexes were exaggerated and planter response was extensor. Fundoscopy examination showed papilloedema. Other systemic examinations were normal.

Relevant investigations were done, where ' $\mathrm{N}$ ' had high ESR $\left(90 \mathrm{~mm}\right.$ in $\left.1^{\text {st }} \mathrm{hr}\right)$, normal total count, differential count and platelet count. Urinary total protein was $1.5 \mathrm{gm} / 24 \mathrm{hr}$. Blood and urine culture showed no growth. Random blood sugar, serum electrolytes and serum calcium were found within normal range. ANA and Anti-dsDNA were positive and serum $C_{3}$ and $C_{4}$ were low. Lipid profile showed mildly raised serum cholesterol ( $210 \mathrm{mg} / \mathrm{dl})$, normal HDL (47 mg/dl), raised LDL (140 mg/dl) and triglyceride (111 mg/dl). Liver function and renal functions were normal. Coomb's test was negative. Chest X-ray, ultrasonography of abdomen and echocardiography were normal. CT scan of brain showed presence of symmetrical diffuse edema predominantly in the occipital regions of the brain (Fig.-1). Considering the history, clinical findings and investigations, ' $N$ ' was diagnosed as a case of Systemic lupus erythematosus with PRES (Posterior Reversible Encephalopathy Syndrome). Initially she was managed in ICU set up with supportive medical treatment by IV fluid, injection manitol, injection phenobarbitone, injection ceftriaxone and antihypertensive drugs (nifedipine, prazosine, captopril) along with occasional sublingual nifidipine. As ' $N$ ' had massive proteinuria, a kidney biopsy was planned, 
but parents did not agree to it. So, specific treatment was started considering the diagnosis as lupus nephritis (? Grade) with PRES. Specific treatment was started by giving injection methyl prednisolone $30 \mathrm{mg} /$ $\mathrm{kg}$ for 3 consecutive days followed by oral prednisolone $2 \mathrm{mg} / \mathrm{kg} /$ day in 4 divided doses. I $\mathrm{V}$ cyclophosphamide pulses $1 \mathrm{gm} / \mathrm{m}^{2}$ monthly was also started. Hydroxychloroquine, calcium with vitamin D supplementation and folic acid was added. Follow up CT scan findings of ' $N$ ' before discharge showed no residual abnormality (Fig.-2). Till now ' $N$ ' had 3 doses of pulse monthly cyclophosphamide therapy. Oral prednisolone is gradually being tapered. Now she is stable and continuing her treatment.

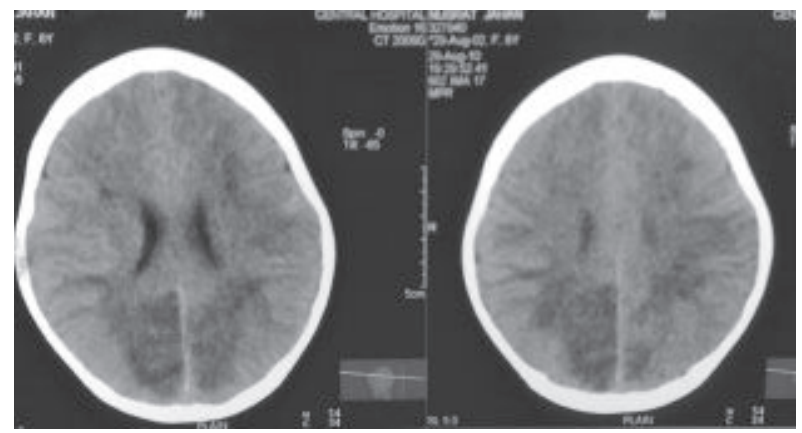

Fig. 1: CT scan of brain showing diffuse hypodensity in occipital region

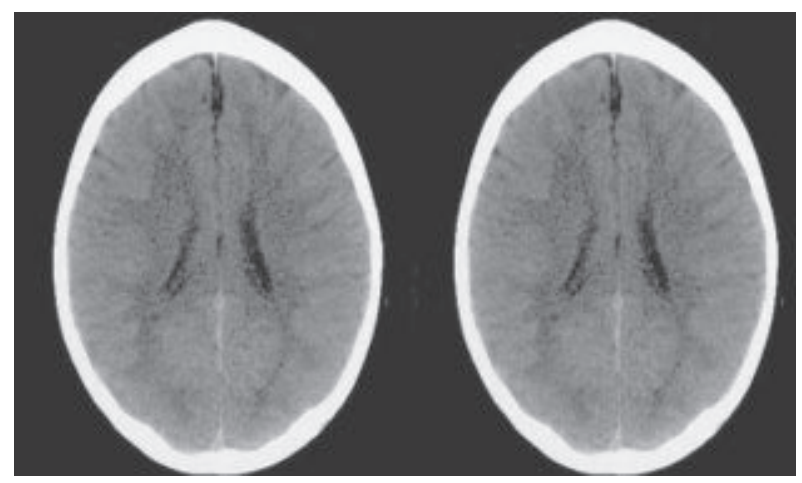

Fig. 2: Follow up CT scan of brain showing normal findings

\section{Discussion:}

Posterior reversible encephalopathy syndrome (PRES) is a clinico-radiological condition characterized by headache, altered mental status, seizure and visual dysfunction and transient motor deficit ${ }^{7}$. It was first described by Hinchey et al in 1996 as reversible posterior leucoencephalopathy. The term PRES was introduced in 2000 as it was found to affect both white matter and grey matter ${ }^{8}$. The pathophysiology of
PRES involves cerebral edema with diffusion of plasma proteins and cells into the extra cellular space. Two distinct theories have been proposed: the vasogenic theory and cytotoxic theory. The cytotoxic theory is that a sudden and severe increase in blood pressure causes cerebral vasoconstriction with cerebral ischemia, hypoxic endothelial cell damage, vasospasm and cytotoxic edema formation ${ }^{9,10}$.

The reversibility of the lesions with rapid treatment argues against this hypothesis. The vasogenic theory suggests that high blood pressure results in impaired cerebral auto-regulations, leading to cerebral vasodilatations, increased vascular permeability, disruption of the blood/brain barrier and vasogenic cerebral oedema. The pathogenesis of PRES in the lupus patients is probably due to multiple factors including lupus disease activity, hypertension, nephritis, and/or medications. Our patient ' $N$ ' had lupus disease activity (evidenced by high anti dsDNA titre and low C3 and C4 level), hypertension and evidence of lupus nephritis (raised urinary total protein).

Conditions commonly associated with PRES include hypertensive encephalopathy, pre-eclampsia, eclampsia, renal failure, transfusion, cytotoxic drugs as well as SLE and other connective tissue diseases ${ }^{11,12}$. Because of the multi-system involvement of SLE, several associated causes may lead to PRES in SLE patients. The main presenting symptoms of PRES are headache, altered mental status, seizures (usually generalized), and visual loss, with an acute to sub acute neurological presentation. Altered consciousness leads to coma in extreme cases. High blood pressure is frequent, but not always present ${ }^{1,13}$. Our patients had headache, convulsion, unconsciousness and loss of vision. She had high blood pressure, papilloedema and upper motor neuron signs. In addition to the clinical presentation, diagnosis of PRES is supported by CT scan findings showing diffuse hypo density in parieto-occipital region ${ }^{14}$. CT scan findings of ' $N$ ' was also consistent with documented findings.

Treatment of PRES must be started as soon as possible. BP should be rapidly controlled with parenteral anti-hypertensive medications with a close monitoring of BP. Close monitoring is needed to prevent hypoperfusion and worsening of the cerebral damage ${ }^{3}$. When PRES is a manifestation of lupus disease activity, with or without active lupus nephritis, intravenous methylprednisolone and cyclopho- 
sphamide are recommended ${ }^{1}$. We have treated "N" accordingly.

PRES is being increasingly recognized in SLE patients may be due to increasing awareness of the condition. This condition must be recognized early, because of potential reversibility with prompt antihypertensive therapy.

\section{References:}

1. Kur JK, Esdaile JM, Posterior Reversible Encephalopathy Syndrome - an underrecognized manifestation of systemic lupus ereythematosus. J Rheumatol 2006; 33: 217883.

2. Hinchey J, Chaves C, Appignani B. A reversible posterior leukoenchephalopathy syndrome. N Eng J Med 1996; 334: 494-500.

3. Karoui KE, Quintrec ML, Dekeyser E, Servais A, Hummel A, Fadel F, et al. Posterior reversible encephalopathy syndrome in systemic lupus erythematosus. Nephrol Dial Transplant 2007;10: 1-7.

4. Singhi P, Subramanian C, Jain V, Singhi S, Ray M. Reversible brain lesions in childhood hypertension. Acta Paediatr 2002; 91(9): 10057.

5. Magnano MD, Bush TM, Herrera I, Aitman RD. Reversible posterior leukoencephalopathy in patients with systemic lupus erythematosus. Semin Arthritis Rheum 2006; 35: 396-402.

6. MakA, Mok CC, Chu CC, To CH, Wong SN, Au TC. Renal damage in systemic lupus erythematosus: a comparative analysis of different age group. Lupus 2007; 16(1): 28-34.
7. Hinchey J, Chaves C, Appignani B, Breen J, Pao $L$, Wang $A$, et al. A reversible posterior leukoencephalopathy Syndrome. N Eng J Med 1996; 334:494-500.

8. Stott VL, Hurrell MA, Anderson TJ. Reversible posterior leukoencephalopathy syndrome: a misnomer reviewed. Intern Med J 2005; 35:8390.

9. Brubaker LM, Smith JK, Lee YZ. Hemodynamic and permeability changes in posterior reversible encephalopathy syndrome measured by dynamic susceptibility perfusion-weighted MR imaging. Am J Neuroradiol 2005;26(4): 825-30.

10. Covarrubias DJ, Luetmer PH, Campeau NG. Posterior reversible encephalopathy syndrome: Prognostic utility of quantitative diffusion-weighted MR images. Am Neuroradiol 2002; 23(6):103848.

11. Kastrup O, Maschke M, Wanke I. Posterior reversible encephalopathy syndrome due to severe hypercalcemia. J Neurol 2002; 249(11): $1563-6$.

12. Ahn KJ, You WJ, Jeong SL. Atypical manifestation of reversible posterior encephalopathy syndrome: finding on diffusion imaging and ADC mapping. Neuroradiology 2004; 46(12): 978-83.

13. Ozyurek H, Oguz G, Ozen S. Reversible posterior encephalopathy syndome: report of three cases. J Child Neurol 2005; 20(12): 990-3.

14. Primavera A, Audenino D, Mavilio N, Cocito L. Reversible posterior leucoencephalopathy syndrome in systemic lupus and vasculitis. Ann Rheum Dis 2001; 60(5): 534-737 